\title{
Noisy Medical Image Enhancement based Dyadic Wavelet
}

\author{
Zhenghong Huang ${ }^{1}$ and $\mathrm{Li} \mathrm{Xia}^{2}$ \\ ${ }^{1}$ Chongqing Engineering Laboratory for Detection, Control and Integrated System \\ ${ }^{1}$ School of Computer Science and Technology, Chongqing Technology and Business \\ University. \\ ${ }^{2}$ School of mathematics and statistics, Chongqing Technology and Business \\ University, 19 Xuefu Avenue in Nan'an, Chongqing, 400067, China \\ ${ }^{1} h z h @ c t b u . e d u . c n,{ }^{2} x l @ c t b u . e d u . c n$
}

\begin{abstract}
We introduce in the paper a method for completing Magnetic Resonance Image(MRI) feature processing by multiresolution representations. The image is decomposed using dyadic wavelet transformation. A new threshold is proposed by way of different thresholds according to the different scales of wavelet coefficients to deal with the wavelet coefficients. The nonlinear enhancing operator is applied for wavelet coefficients with the enhancement method in the corresponding scales. Our results show that the proposed method outperforms conventional enhancement method, the information entropy and contrast improvement index of the images is improved, enhances edge texture of images.
\end{abstract}

Keywords: Dyadic wavelet transform, Multiresolution representations, Threshold function, Image enhancement

\section{Introduction}

In the process of image acquisition and transmission, the image is easily contaminated by noise which always to affect the correct analysis, understanding and application of the image. Image enhancement is one of the important methods of image preprocessing, which can improve the visual effect of the image. Thus makes the enhanced image features are more prominent.

Currently, most of denoising approaches are based on spatial domain and frequent domain. However, in spatial domain enhanced image processing methods, such as unsharp masking method, better contrast enhancement can be achieved for images without noise, while for noisy image, the noise is enhanced while enhancing the detail features[1]. In the frequency domain processing method of image enhancement, by image enhancement algorithm based on multi-scale transform, such as selecting the appropriate threshold, the high-frequency coefficients are distinguished and adopted different strategies to enhance or suppress them. However, when there is little of prior knowledge of noisy images, it is difficult to choose the appropriate threshold [2]. Although it has been improved[3], too many thresholds are estimated in threshold function, and it is more difficult to achieve. At the same time, the sampling method is proposed to suppress the translation invariant of the wavelet transform[4], which leads to the small transfer of the digital signal in the frequency domain, which results in the Pseudo-Gibbs phenomenon.

Received (July 13, 2017), Review Result (November 14, 2017), Accepted (November 26, 2017) 
Wavelet analysis has the characteristics of time frequency localization and multiresolution. In particular, the dyadic wavelet transform has translation invariance compared with the biorthogonal wavelet transform. It can be applied in image decomposition to obtain complete high frequency components, which is suitable for image enhancement, edge detection and feature extraction, etc.

In this paper, we propose an enhanced algorithm of image, which based on dyadic wavelet transform and nonlinear operator of redundancy multiresolution. The image is decomposed different localized subbands by multiresolution representation of dyadic wavelet. A threshold function based on the dyadic wavelet is proposed, and a wavelet thresholding method based on the improved threshold function is employed to reduce image noise by these different bands, the threshold and threshold function. Then, the image is enhanced by filters and then is decomposed into different bands[5,6]. The experiment results indicate that they can maintain the texture and details of the MRI images while removing the noise[7,8].

\section{Multiresolution Representations of the Dyadic Wavelet Transform}

Analysis from two-dimensional signal, tensor space $\left\{V_{j}^{2}: V_{j} \otimes V_{j}\right\}_{j \in Z}$ makes up the multiresolution analysis of $L^{2}\left(R^{2}\right)$, setting $\psi(x)$ as one-dimensional scaling function, $\varphi(x)$ the corresponding dyadic wavelet, thus an orthonormal basis of $L^{2}\left(R^{2}\right)$ is defined

$$
\psi(x, y)=\psi(x) \psi(y) \quad(x, y) \in R^{2}
$$

three two-dimensional dyadic wavelet are available

$$
\begin{aligned}
& \varphi^{(1)}(x, y)=\psi(x) \varphi(x) \\
& \varphi^{(2)}(x, y)=\psi(y) \varphi(x) \\
& \varphi^{(3)}(x, y)=\varphi(x) \varphi(y)
\end{aligned}
$$

Two-dimensional dyadic wavelet can be expressed as

$$
\varphi_{2^{j}, m, n}^{(i)}(x, y)=\frac{1}{\sqrt{2^{j}}} \varphi^{(i)}\left(\frac{x-n}{2^{j}}, \frac{y-m}{2^{j}}\right), \quad j, n, m \in Z, j \geq 0, i=1,2,3
$$

According to two-dimensional multiresolution analysis, low-frequent separation of twodimensional image signal $a_{j}(x, y)$ under the scale $2^{j}$ can be showed as the two-dimensional sequence

$$
\begin{aligned}
& a_{j}[n, m]=\left(f(x, y), \psi_{2^{j}, m, n}(x, y)\right) \\
& =\frac{1}{\sqrt{2^{j}}} \iint_{R^{2}} f(x, y) \psi\left(\frac{x-n}{2^{j}}, \frac{y-m}{2^{j}}\right) d x d y
\end{aligned}
$$

The high frequent components are showed respectively as

$$
\begin{gathered}
c_{j}^{1}[n, m]=\left(f(x, y), \varphi_{12^{j}, m, n}(x, y)\right)= \\
\frac{1}{\sqrt{2^{j}}} \iint_{R^{2}} f(x, y) \varphi^{(1)}\left(\frac{x-n}{2^{j}}, \frac{y-m}{2^{j}}\right) d x d y \\
c_{j}^{2}[n, m]=\left(f(x, y), \varphi_{22^{j}, m, n}(x, y)\right) \\
c_{j}^{3}[n, m]=\left(f(x, y), \varphi_{32^{j}, m, n}(x, y)\right)
\end{gathered}
$$

from àtrous algorithm [9]

$$
a_{j+1}[n]=\sum_{k} h[k] a_{j}\left[n+2^{j} k\right] \quad j=0,1,2 \ldots . J
$$




$$
\begin{array}{ll}
c_{j+1}[n]=\sum_{k} g[k] a_{j}\left[n+2^{j} k\right] & j=0,1,2 \ldots J \\
a_{0}[n] & =\int_{-\infty}^{+\infty} f(t) \varphi(t-n) d t
\end{array}
$$

where $h$ is the low-pass filter corresponding to the scaling function $\psi(x), g$ is the the high-pass filter corresponding to dyadic wavelet function $\varphi(x)$.

The image signal is decomposed into a low frequent component and three high frequent components, $a_{j}$ is the low-frequent component of scale $2^{j}, c_{j}^{1}, c_{j}^{2}, c_{j}^{3}$ are the vertical, horizontal, diagonal high frequent components of scale $2^{j}$ respectively, low frequent component $a_{j}$ can still be decomposed according to above way. The original signal $a_{j}[n]$ $(j=0,1,2 \ldots . J)$ can be reconstructed by the dyadic wavelet signals $a_{j+1}[n]$ and $d_{j+1}[n]$ in the decomposition equation and showed as

$$
\begin{aligned}
a_{j}[n]=\frac{1}{2}\left\{\sum_{k} \tilde{h}[k] a_{j+1}\left[n-2^{j} k\right]+\right\} & \\
& \left.+\sum_{k} \tilde{g}[k] C_{j+1}\left[n-2^{j} k\right]\right\} \quad j=1,2 \ldots . . J
\end{aligned}
$$

From dyadic wavelet filter

$$
\sum_{k} h[k]=1, \quad \sum_{k} g[k]=0
$$

and fast àtrous algorithm, the fast decomposition of separable dyadic wavelet is realized, the concrete steps are as follows.

(1). Decomposing the two-dimensional image signal into image components within different frequent bands and transforming it into wavelet separately by its rows columns.

(2). Wavelet decomposition on each layer decompose image in to four equal size subband images, whose numbers of dyadic wavelet coefficients decomposing on different scales are equal to the numbers of pixels of the original image.

(3). In this way, approaching signals and continuing decomposition by analogy.

\section{Threshold and Threshold Function}

Image after wavelet transform, the corresponding image feature coefficient amplitude is larger, and has a strong correlation between neighboring scales, and the corresponding noise coefficient amplitude is relatively small, the correlation between the adjacent scales is not strong, using this property can be more convenient in image characteristics and noise.

The denoising algorithm proposed by Donoho method is based on the orthogonal wavelet transform, because the orthogonal wavelet transform does not have the inverse transformation. So the denoising results will appear visual distortion. A new threshold function is adopts to deal with the wavelet coefficients[10,11].

The main information of noise image by dyadic wavelet transformation is distributed in the low frequent region, the noise and image details are mainly distributed in the high frequent region, most image information is also distributed in a small number of wavelet coefficients, whose noise has the same amplitude. According to different scales, different threshold values can be chosen for wavelet coefficients denoising. 
In order to reflect the feature that the standard deviation of noise in different decomposition scale varies with scale increasing in wavelet multi-scale analysis, assume the different lever threshold of dyadic wavelet are

$$
\delta_{i}=\sigma \sqrt{2 \ln N} / \ln \left(e+2^{i-1}-1\right)
$$

where $\sigma$ is the standard deviation of noise and $\mathrm{N}$ is the image pixels. When scale $\boldsymbol{i}=\mathbf{1}$, it becomes the original formulation. When $i>1$, the threshold $\delta$ decrease with the scale increase, and the noise amplitude decrease with the scale increase. Moreover, adapt the decomposition noise in different scale and the lower bound of threshold denoising to propose the improved lever threshold function.

To the noisy mixed signal, denoise the measured signal fist, that is, choose a threshold for the noisy signal wavelet and then process the threshold to the wavelet transformed scale discrete detail to signal denoise

$$
\hat{\omega}_{j, k}= \begin{cases}\operatorname{sgn}\left(\omega_{j, k}\right)\left\{\omega_{j, k}-\frac{\delta_{j}}{\exp \frac{\omega_{j, k} \mid-\delta_{j}}{\delta_{j}}}\right\} & \left|\omega_{j, k}\right| \geq \delta_{j} \\ 0 & \left|\omega_{j, k}\right|<\delta_{j}\end{cases}
$$

where $\omega_{j, k}$ is the wavelet coefficient, $\hat{\omega}_{j, k}$ is the estimated wavelet coefficient and $\delta_{j}$ is the threshold concerned scale[12]. When $\left|\omega_{j, k}\right|>\delta_{j}$, the difference between $\hat{\omega}_{j, k}$ and $\omega_{j, k}$ is small and the reconstructed signal is approximate real signal better on scale $j$.

The mathematic model of noisy image on scale $i$ is

$$
y_{i}=x_{i}+\delta n_{i} \quad i=1,2 \cdots \cdots N
$$

where $y_{i}$ is the noisy image, $x_{i}$ is the denoising signal, $n_{i}$ is the independent identically normal distribution random noise, $N$ is the number of pre-pixels.

By adapting different thresholds under various conditions of scale, so that the threshold function can automatically adjusted. To improve the denoising accuracy of new threshold function, the denoising error lower bound of the new threshold.

\section{Nonlinear Enhancement Function of the Dyadic Wavelet}

Set the basic wavelet function $\varphi(x) \in L^{2}(R)$ to meet

$$
\int_{R} \frac{|\hat{\varphi}(\omega)|^{2}}{|\omega|} d \omega<\infty
$$

where $\hat{\varphi}(\omega)$ is the Fourier transform of $\varphi(x)$, there is

$$
\varphi_{a, b}(t)=\frac{1}{\sqrt{a}} \varphi\left(\frac{x-b}{a}\right)
$$

signal function $f(t) \in L^{2}(R)$, whose wavelet transform function is expressed as

$$
(W f)(a, b)=W_{a} f(b)=\int f(t) \frac{1}{\sqrt{a}} \varphi *\left(\frac{x-b}{a}\right) d t=\left(f, \varphi_{a, b}\right)
$$

where $a$ is the scale parameter, $b$ is the displacement parameters.

Discretion the scale parameter, letting $a=2^{-j}$, Equation (1) can be written as 


$$
W_{2^{j}} f(x)=f(x) * \varphi_{2^{j}}(x)=\int_{R} f(t) \frac{1}{\sqrt{a}} \varphi_{2^{j}}(x-t) d t
$$

then dyadic wavelet transform of $f(x)$ can be expressed as

$$
f(x)=\sum_{j=-\infty}^{\infty} W_{2^{j}} f(x) * \chi_{2^{j}}(x)=\sum_{j=-\infty}^{\infty} f(x) * \varphi_{2^{j}}(x) * \chi_{2^{j}}(x)
$$

where wavelet $\varphi(x)$ and $\chi(x)$ satisfy the condition.

Letting

$$
\sum \hat{\varphi}_{2^{j}}(x) * \hat{\chi}_{2^{j}}(x)=1
$$

where $\hat{\varphi}(\omega)$ and $\hat{\chi}(\omega)$ are the transforms of Fourier respectively.

Setting scale function $\phi(x)$, whose Fourier is transformed as

$$
|\hat{\phi}(x)|^{2}=\sum_{j=1}^{\infty} \hat{\varphi}\left(2^{j} \omega\right) \hat{\chi}\left(2^{j} \omega\right)
$$

Meeting the telescopic equation

$$
\hat{\phi}(x)=H\left(\frac{\omega}{2}\right) \hat{\phi}\left(\frac{\omega}{2}\right)
$$

where $H(\omega)$ is the Fourier transform of discrete filters.

Letting

$$
\begin{aligned}
& \hat{\psi}(\omega)=G\left(\frac{\omega}{2}\right) \hat{\phi}\left(\frac{\omega}{2}\right) \\
& \bar{\chi}(\omega)=K\left(\frac{\omega}{2}\right) \hat{\phi}\left(\frac{\omega}{2}\right)
\end{aligned}
$$

where $G(\omega)$ and $K(\omega)$ are discrete filters.

To achieve the purpose of enhancement, letting $\hat{\psi}(x)=-\omega \hat{\theta}(\omega)$, in the frequent domain where $\theta(\omega)$ repents low-pass filter, there is

$$
\hat{\theta}(\omega) \neq 0, \quad \lim _{\omega \rightarrow \infty} \hat{\theta}(\omega)=0
$$

According (7), the following discrete filters can be constructed

$$
\begin{aligned}
H(\omega)= & {\left[\cos \frac{\omega}{2}\right]^{2 n} } \\
G(\omega)= & {\left[\sin \left(\frac{\omega}{2}\right)\right]^{2} } \\
& K(\omega)=\frac{1-|H(\omega)|^{2}}{G(\omega)}=-\frac{1}{4} \sum_{k=0}^{2 n-1}\left[\cos \left(\frac{\omega}{2}\right)\right]^{2 l}
\end{aligned}
$$

From (5)-(8), it is available

$\hat{\phi}(\omega)=[\sin (\omega / 2) / \omega / 2]^{2 n}$

in the frequent domain, meeting

$\hat{\psi}(\omega)=-\omega^{2} \hat{\theta}(\omega)$

$\hat{\chi}(\omega)=-\beta(\omega)$

equation (3) can be represented as

$W_{2^{k}} f(x)=2^{k} \frac{d^{2}}{d x^{2}}\left[f(x) * \theta_{2^{k}}(x)\right]$ 
By the method of nonlinear enhancement, the direct enhancing function $\tilde{f}(x)$ is available through enhancing the channel $p$ in the time domain

$$
\begin{aligned}
\hat{f}(x)=\sum_{k=-\infty}^{+\infty} E\left[W_{2^{k}} f(x)\right] * \chi_{2^{k}}(x) & \\
= & f(x)+\left\{E\left[2^{p} \frac{d^{2}}{d x^{2}}\left(f(x) * \theta_{2^{k}}(x)\right]-2^{p} \frac{d^{2}}{d x^{2}}\left[f(x) * \theta_{2^{p}}(x)\right]\right\} * \chi_{2^{p}}(x)\right.
\end{aligned}
$$

$\widehat{f}(x)$ is simplified as

$$
\hat{f}(x)=f(x)-\left[E\left(2^{p} \frac{d^{2} f}{d x^{2}}\right)-2^{p} \frac{d^{2} f}{d x^{2}}\right]
$$

Where the function of soft edge model is defined by

$$
f(x)=\frac{1}{1+e^{-\alpha x}}
$$

Its second derivative

$$
f^{\prime \prime}(x)=-(\alpha / 2)^{2} \sinh (\alpha x / 2) \cosh (\alpha x / 2)^{-3} \text { is antisymmetric. Enhancement }
$$

function $\mathrm{E}(\mathrm{x})$ is monotonicity and continuity.

\section{Image Enhancement}

As describe as above, based on the nonlinear operator, our approach can not only retain texture and detail of image but also reduce the noise of image [13].

(1). Compute the 2-D $\operatorname{WTf}(m, n)$ of noise image $f(m, n)$ and decompose $N \times N$ images into four so that the energy concentrates on few coefficients.

(2). Choose suitable wavelet and then perform a wavelet transformation with decomposition level $\mathbf{j}$. We get the wavelet coefficients. The denoised image $f_{T}(m, n)$ is obtained based on threshold and the wavelet coefficients $C_{f}$ are obtained. The enhanced image $f^{-1}(m, n)$ is computed by inverse Discrete Wavelet Transform (IDWT) of $C_{f}$.

(3). Based on nonlinear enhancement process for the high frequency sub-image, the obtained wavelet coefficients can reconstruct the image to enhance the detail of

image. By the method describe above, output image $\hat{f}(m, n)$ is obtained.

\section{Experimental Results and Analysis}

We analysis the performance of image enhancement based on brain MRI image [14]. The MRI image obeys Rician distribution, whose noise is the Rician noise of non-zero mean value, whose average normally associated with partial strength of image, which is regarded as obeying to Gaussian distribution in the process of MRI. We conduct emulative experiment under the same thresholds (1), and test the MR brain image, whose noise standard error is $\sigma=18$, and decomposed scale as 4 is taken. For thresholds $\delta_{j}$, where $\mathrm{j}$ is decomposed length, $N$ is signal length.

As shown in original image, the contrast is low and the edge of tissues is vague. Compared with the original image, the over bright region is weakened, the dark region is enhanced, and the edge contour and detail information are enhanced to be clearer. The flat region of the image is blurred by Unsharp Masking(UNS) enhancement algorithm. 
In our experiment, the information entropy and contrast are employed to estimate the performance of the proposed method. The entropy is often used as a characterization of the information content of a data source and defined as follows:

$$
E=-\sum_{k=1}^{255} p_{k} \log _{2}\left(p_{i}\right)
$$

where $p_{i}$ is the probability of the gray-scale $i$, the contrast is treated as the clarity. If the contrast is lager, the image is sharpness.

A quantitative measure of contrast improvement can be defined by a Contrast Improvement Index (CII),

$$
C I I=C_{\text {Processed }} / C_{\text {Original }}
$$

where $C_{\text {Processed }}$ and $C_{\text {Original }}$ are the contrasts for a region of the processed and original images, respectively. For a given image, we divide it into $3 \times 3$ patch and $C$ are the average contrast of all patches. The contrast of each patch is defined as

$$
C=(\max -\min ) /(\max +\min )
$$

where max is the mean gray-scale value of a surrounding region, called the maximal graylevel value and min denotes its minimal gray- level value. This definition of contrast has the advantage of being independent of the actual rang of gray level in the image.

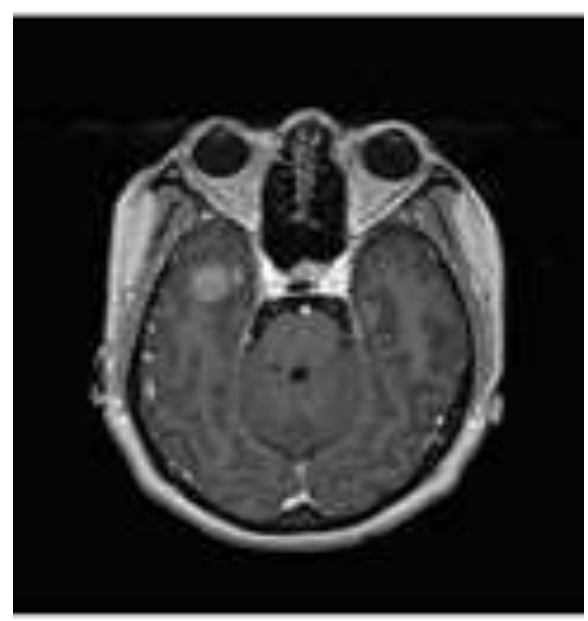

(a) Original Image of Brain Cancer

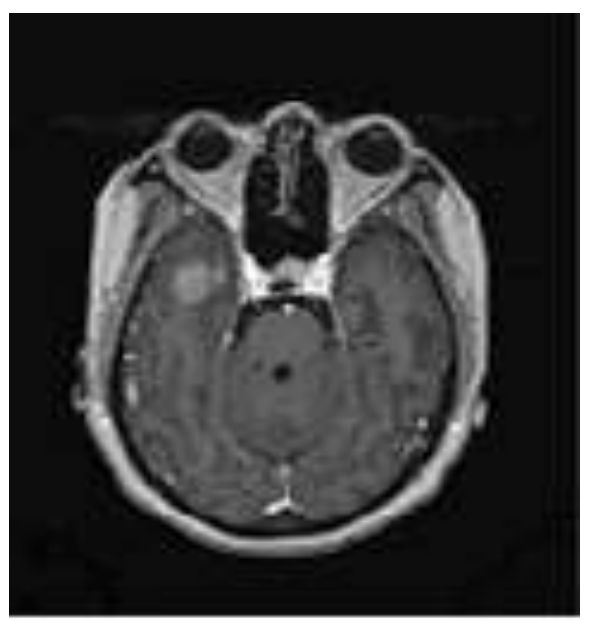

(b) Unsharp Masking Method 


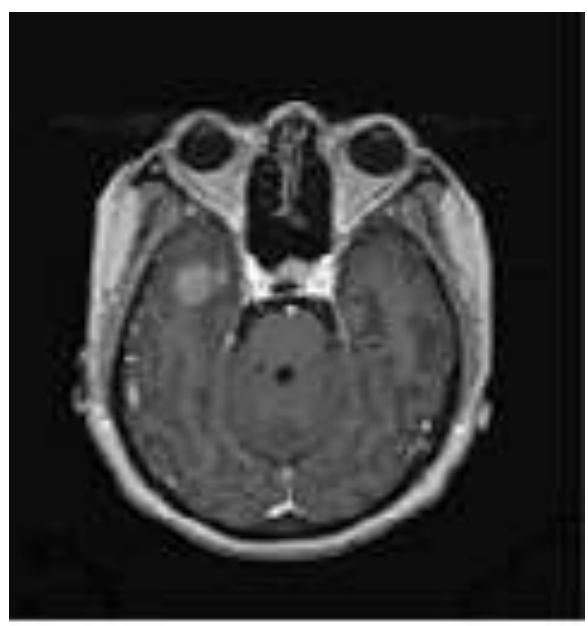

(c) The Paper Method

\section{Figure 1. Comparing the Performance of Various Methods on Brain MRI Image}

In experiment, the two evaluation indexes are adopted by UNS enhancement and the method. We select a MR brain image with size of $256 \times 256$. Figure 1(a) shows the original image. From which, we can be seen that it contain more noise; Figure 1(b) shows the enhancing image by UNS method, Figure 1(c) shows the enhancing image based on the proposed approach. The data of MR brain image is prepare as follows:

Table 1. The Comparison of Enhancement Performance

\begin{tabular}{|l|l|l|l|}
\hline \multicolumn{1}{|c|}{ Category } & $\begin{array}{l}\text { Original } \\
\text { image }\end{array}$ & UNS method & $\begin{array}{l}\text { The paper } \\
\text { method }\end{array}$ \\
\hline $\begin{array}{l}\text { Information } \\
\text { entropy }\end{array}$ & 2.4842 & 2.5968 & 3.3931 \\
\hline $\begin{array}{l}\text { Contrast } \\
\text { improvement index }\end{array}$ & 1.1428 & 4.6304 & 6.7139 \\
\hline
\end{tabular}

From Table 1, the information entropy and contrast improvement index of the enhanced image are greatly improved compared with UNS enhancement. Table 1 shows the contrast values and CII for the MR brain image features in the Figure 1. Nonlinear enhancement of dyadic wavelet coefficient performed significantly better than (UNS).

The information entropy is used to evaluate quality of image. For a gray scale image, if the entropy of its enhancing image is large, it implies there are more details in the enhancing image and its information capacity is large. Figure 1(c), we can see that the image is effectively enhanced and contains more distinguishing texture.

The experimental results show the entropy and contrast of enhancement image are improved significantly and the proposed approach boosts the feature information of image edge.

\section{Conclusion}

In this paper, the dyadic wavelet based nonlinear enhancing approach can capture more detail of image. By àtrous method and dyadic wavelet transform, the image is decomposed into four subbands with different scale and the main energy concentrates few wavelet coefficients. The nonlinear enhancement of dyadic wavelet multiscale representations is 
employed for MRI denoising, image enhancement and image edge processing. The proposed approach not only enhances the local detail but also suppresses the noise. In addition, by reducing noise in different bands, our method can enhance edge features of lesion and improve the accuracy of diagnosis.

\section{Acknowledgements}

This work was supported by the Nature Science Foundation of CEL(No.DCIS20150301).We thank all teachers in our lab for helpful discussion.

\section{References}

[1] X.-K. Xiao and S.-F. Li, "Edge-preserving image denoising method using urvelet transform", Journal of China institute of communications, vol.25, no.2, (2004), pp. 9-15.

[2] D L Donoho, "De-nosing by soft-thresholding", IEEE Trans Inform Theory, vol.41, no.3, (1995), pp. 613627.

[3] H. Y. Gao and A. G. Bruce, "WaveShrink with firm shrinkage", Statistica Sinica., oal.7, no.7, (1997), pp. 855-74.

[4] I. Kohen and A. Laine, "A discrete dyadic wavelet transform for multidimensional feature analysis", In Time Frequency and Wavelet Transform in Biomedical Engineering, IEEE Press, (1998), pp. 425- 449.

[5] A. Laine and S. Song, "Wavelet processing techniques for digital mammography", In proceedings of SPIE: Conference on Visualiztion in Biomedical Computing, Chapel Hill, NC, (1992), pp.13-16.

[6] A. Laine, "Multiscale wavelet representations for mammographic feature analysis", In Image Enhancement techniques: Computer Science, National Cancer Institute Breast Imaging Workshop: Stat of the Art and New Technologies, Bethesda, MD, (1991).

[7] G.-A.Hossien-Zadeh, H.Soltanian-Zaseh and B.A. Ardekani, "multiresolution fMRI activation detection using translation invariant wavelet transform and statistical analysis based on resampling", IEEE Trans.Med.Imag., vol.22, no.3, (2003), pp. 302-314.

[8] R. Willett and W. Philips, "Platelets: A multiscale approach for recovering edges and surface in photon limited medical imaging”, IEEE Trans.Med Imag., vol.22, no.3, (2003), pp.332-350.

[9] Laine and S. Song, "Wavelet processing techniques for digital mammography", In Procedings of SPIE:conference on Visualization in Biomedical Computing, Chapel Hill, NC, (1992), pp. 13-16.

[10] K. Berkner, O. Raymond and J.R. Wells, "Smoothness estimates for soft-threshold denoising via translationinvariant wavelet transforms", Applied and Computational Harmonics Analysis, vol.1, no.12, (2002), pp.124.

[11] M. allat S. Zhong S," Characterization of signals from multiscale edges", IEEE Transactions on Pattern Analysis \& Machine Intelligence, vol.14, no.7, (1992), pp. 710-732.

[12] Z. Huang and B. Fang, "Image denoising based on the Dyadic wavelet transform and improved threshold", Wavelets, multiresolution and information processing, vol.7, no.3, (2009), pp. 269-280.

[13] T. Abukirim, S. Yakano and K Niijima, "Fast face detection by lifting dyadic wavelrt filters", Procedings of the IEEE International Conference on Image Processing, ICIP2003, (2003),pp. 893-896.

[14] A. Macovski, "Noise in MRI", Magnetic Resonance in Medicine, vol. 36, no. 3, (1996), pp.494-497.

\section{Authors}

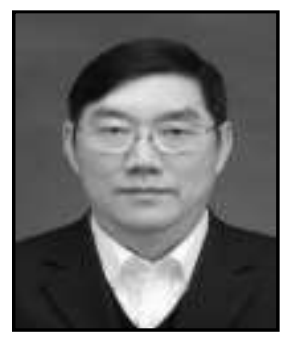

Zhenghong Huang, he is a professor of computer science and information engineering at Chongqing Technology and Business University. His research interests include image processing, pattern recognition, wavelet analysis and application.

Email: hzh@ctbu.edu.cn

Phone: 862362768938 
International Journal of Signal Processing, Image Processing and Pattern Recognition Vol. 11, No. 1 (2018)

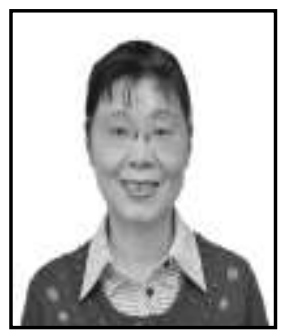

Li Xia, she is a professor of mathematics and statistics at Chongqing Technology and Business University. Her research interests include image processing, data mining. Email: xl@ctbu.edu.cn 